\title{
Preparation and Characterization of CdSe Single Crystal
}

\author{
A. Abd - El Mongy \\ Physics department, Faculty of science, Helwan University, \\ Cairo, Egypt
}

\begin{abstract}
High quality of CdSe single crystal is grown from the melt. X-ray fluorescence (XRF) showed that the ingot crystal has Cd:Se ratio very close to 1 . The Photoresponse spectra in the energy range 1.2- $2.1 \mathrm{eV}$ is found to change with increasing temperature from $80 \mathrm{~K}$ to $300 \mathrm{~K}$. At temperatures $\leq 200 \mathrm{~K}$ three peaks are observed at 1.45; 1.73 and $1.97 \mathrm{eV}$; whereas above $200 \mathrm{~K}$, the peaks at 1.45 and $1.97 \mathrm{eV}$ are washed out. These peaks are interpreted in terms of the band model, considering the band splitting. The photocurrent-temperature behaviour showed three main regimes: a weak dependence at lower temperatures; thermal quenching region (maximum at $200 \mathrm{~K}$ ) and a marked decrease at higher temperatures. The onsets and offsets of the three regions are found to depend on the incident photon energy. A very high lifetime (milliseconds); being temperature dependent is obtained from the frequency resolved photoconductivity. The data are explained on the bases of recombination kinetics controlled by defect centres.
\end{abstract}

\section{Introduction:}

Among II-VI semiconducting compounds cadmium selenide (CdSe) single crystalline material has attracted significant interest due to their attractive potential applications. For example radiation detector [1\&2], nonlinear optical devices [3], photoluminescence response [4]. However the melting point of $\mathrm{CdSe}$ is very high $\left(1239^{\circ} \mathrm{C}\right)$ and the equilibrium partial pressure of $\mathrm{Cd}$ and $\mathrm{Se}$ along liquidus are also very high [5]. The thermal conductivity of CdSe is very low [6]. These are in fact serious obstacles to the growth of CdSe single crystals. Therefore stoichiometric imperfection, dislocations, second phase precipitates and polycrystalline are still very difficult to avoid during the crystal growth. In order to overcome the defect formation in CdSe single crystal, various methods have been performed [7\&8]. In order to understand the structure and photoelectronic properties, and the need to assess their potential technological applications, several models have been proposed. The bands model [9], excitonic emission [10], donor-acceptor pair recombination [11] and band splitting [12] although exhibit remarkable features, the physical processes causing the photoconductivity kinetics have not been sufficiently investigated.

However up to now, all kinds of the growth methods on the CdSe single crystal have not obtained the result of great significance and only limited 
information is available. Furthermore, the band structure and recombination mechanisms have been a matter of discussion among different authors and a conclusive study seems open until now. Thus growth of CdSe crystals with their characterization is in need of further study and highlights the significance of the present investigation. The present work was focused on preparing good quality of the CdSe single crystal. The as-grown bulk crystal has been characterized by $\mathrm{X}$-ray analysis. Photoconductivity measurements that provide recombination parameters have been also performed over the temperature range $80-300 \mathrm{~K}$.

\section{Experimental details:}

For CdSe crystal growth, the starting material was bought with labeled purity of $99.9999 \%$. Polycrystalline CdSe was ground to powder in an agate mortar, and then loaded into quartz ampoule of $16 \mathrm{~cm}$ length and $12 \mathrm{~mm}$ diameter sealed under $10^{-5}$ Torr. The crystal growth was carried out in a two zone furnace using the classical method of the melt growth (Bridgman technique) in which the ampoule slowly moves at a rate of $1.5 \mathrm{~mm} / \mathrm{h}$ using a puller with a speed-control motor. First the ampoule was held at a temperature of $1250^{\circ} \mathrm{C}$ higher than the CdSe melting point for 24 hours. During the fusion processes the ampoule was frequently agitated, using mechanical system, to ensure homogeneity for the melt. Secondly, the ampoule with its charge was moved to the crystallization region $\left(1050^{\circ} \mathrm{C}\right)$. The temperature was kept constant within $\pm 5^{\circ} \mathrm{C}$ during growth. Duration of eight days is required to move the ampoule through the two zone tube furnace. The as grown CdSe ingot was cleaved by blade and cut into set of samples. The X-ray diffraction spectra, using a Philips PW 1710 diffractometer, showed that the crystallinity of the ingot CdSe was perfect with $\{100\}$ preferred orientation. The stoichiometry (Cd/Te ratio) of the crystal was investigated by X-ray fluorescence (XRF) using Diano X-ray spectrometer with LiF200 crystal and CrKa radiation powered at $5000 \mathrm{kV}, 35 \mathrm{~mA}$; step scanning techniques with step size $(\Delta 2 \theta)$ of $\pm 0.1{ }^{\circ} \mathrm{C}$ and a period of $1 \mathrm{~s}$. In fact the parameters relating weight concentration and line intensity of fluorescence X-rays produce complex functions [13].

The temperature dependence of photoconductivity was investigated using gap cell configuration in which two aluminum electrodes of spacing in between $=3 \mathrm{~mm}$ and dimensions of $1.2 \mathrm{~mm} \times 5 \mathrm{~mm}$ were evaporated on one of the crystal surfaces. The electrodes were deposited using the Edwards (E 306A) coating system at pressure of $2 \times 10^{-5}$ Torr. The thickness of the sample was made smaller than the gap distance to ensure field homogeneity throughout the crystal. A beam of light from a tungsten lamp $(1000 \mathrm{~W})$ was incident on a M4G11 grating monochromator with band width of $1 \mathrm{~nm}$ and focused at right angle onto the crystal surface plane through an optical system consisting of two convex lenses. The sample mounted in an evacuated Oxford cryostat (DN1704) 
was connected in series with DC power supply. Fine wires were attached by silver paste to the holder and the electrodes. The cryostat with its special window keep the temperature of the investigated crystal constant during the measurements to reduce photoconductivity due to heating of the sample during the illumination processes. The ohmic nature of the contacts was checked by measuring the steady current-voltage $(\mathrm{J}-\mathrm{V})$ characteristics in opposite directions to ensure that the photoinjection contribution from the electrodes is neglected. For measuring the frequency dependence of the photoconductivity, the light is collimated on the sample through a mechanical chopper system (Stanford SR540) with chopper controller which gives symmetric square light pulses. The chopping frequency was varied from 5 to $100 \mathrm{~Hz}$. The lifetime of the photocarriers was calculated to understand the photoconductivity kinetics of the material. The variation across a load resistance $\left(10^{7} \Omega\right)$ due to the modulated photocurrent was measured through lock in amplifier SR510 Stanford. The current passing through the specimen was measured using Keithley 610C electrometer. To achieve temperature stability up to $\pm 0.1{ }^{\circ} \mathrm{C}$, an Oxford temperature stabilizer (DTC2) was used. Measurements were carried out in an Oxford (CDN1704) vacuum cryostat evacuated to $2 \times 10^{-4}$ Torr to avoid oxidation at high temperatures and to prevent vapour condensation at low ones.

\section{Results and Discussions:}

The XRF showed that the crystal has a stoichiometry ratio $\mathrm{Cd}: \mathrm{Se}=0.98$ $: 1$, which is close to the ideal stoichiometry ratio of 1 (more details have been given in previous paper [14]). Figure 1 shows the spectral distribution of the CdSe single crystal, measured at constant excitation intensity (500 lux); in the temperature range $80 \mathrm{~K}-300 \mathrm{~K}$ and the incident photon energy changes from 1.2 to $2.1 \mathrm{eV}$. The rapid decrease of photocurrent in the field of high energies highlights the recombination of non-equilibrium charge carriers through surface states. This implies that the intrinsic defects have tendency to segregate at the surface of the crystal. At temperature $\leq 200 \mathrm{~K}$ three bands are obtained, the first centred at 1.45 and the second located at 1.73 and the third around $1.97 \mathrm{eV}$. This indicates that in addition to the fundamental transition, two additive transitions are present. The first band has a Gaussian lineshape with slight asymmetry, typical features of the emission bands due to donor-acceptor pair transitions [15]. It is observed that the peak position and emission band intensity change with temperature. It can be seen also that the observed peak at $1.45 \mathrm{eV}$ is broad and of small intensity. It is likely that some defects produce centres in the band which steal photons and give in turn small photocurrent. The broadening can result from competion between more than one optical transition (each due to defect center) both of which causes photoconduction. Above 200 $\mathrm{K}$, the character of the spectral dependence has changed, only the peak at 1.73 $\mathrm{eV}$ (the non-perturbed energy band of the CdSe) is present and the other two 
bands disappear. Such behaviour is explained in the base of an energetic bands model [16\&17] considering the spin orbit and crystal field band splitting observed recently in the CdSe materials [12]. As Sketched in Fig.2, the $1.45 \mathrm{eV}$ peak could be due to transition from upper branch of the valence band to the minimum of the conduction band with $0.28 \mathrm{eV}$ difference from the fundamental band gap $(1.73 \mathrm{eV})$. The second electronic transition corresponds to energy difference $=0.24 \mathrm{eV}$. Thus the effective band splitting $=0.52 \mathrm{eV}$. The data indicates that the band splitting transitions disappear as the temperature becomes higher than $200 \mathrm{~K}$.
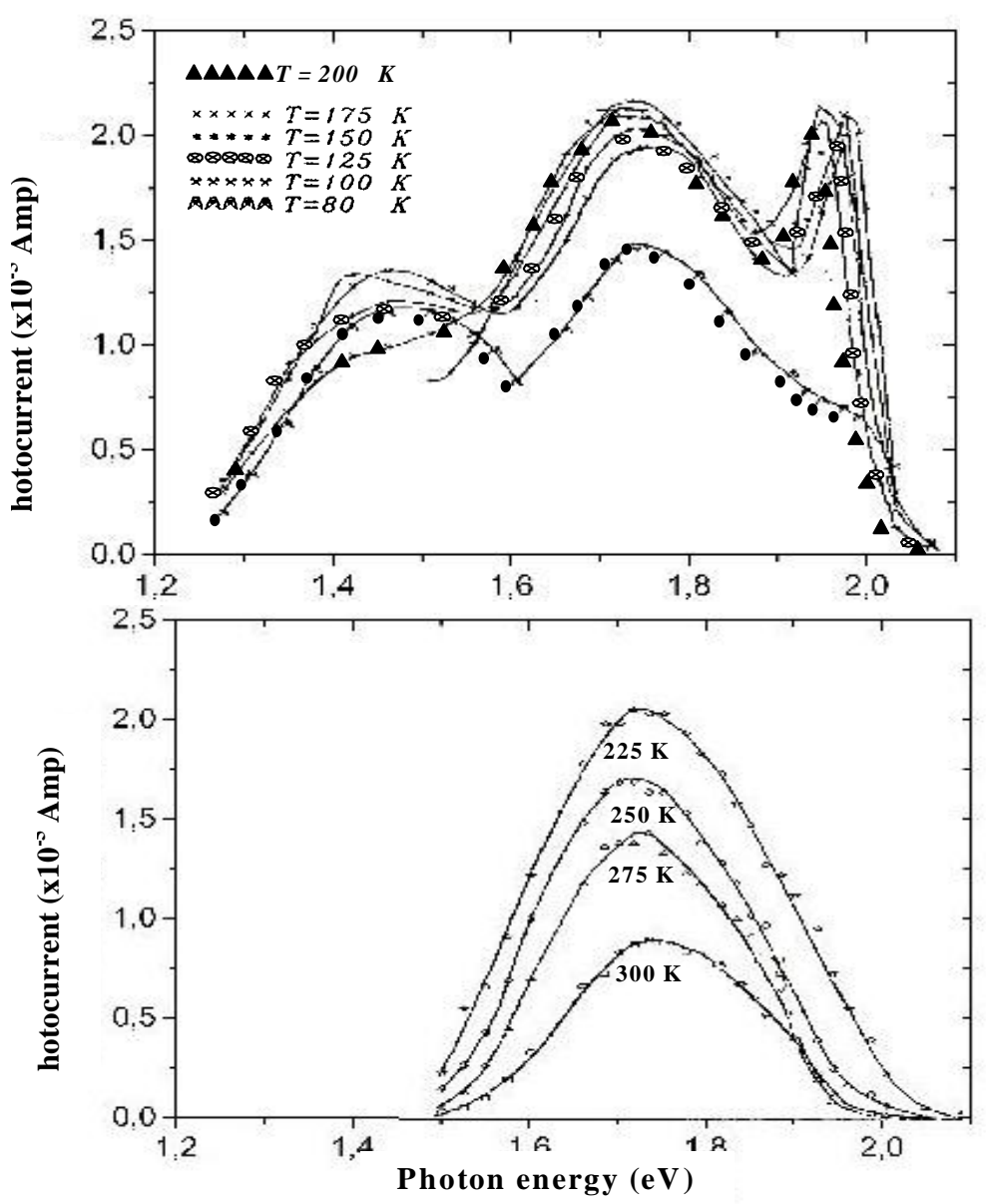

Fig.(1): Spectral dependence of photocurrent in CdSe crystal at bias voltage (15 V); light intensity (500 Lux) and different temperatures (80-300 K). 


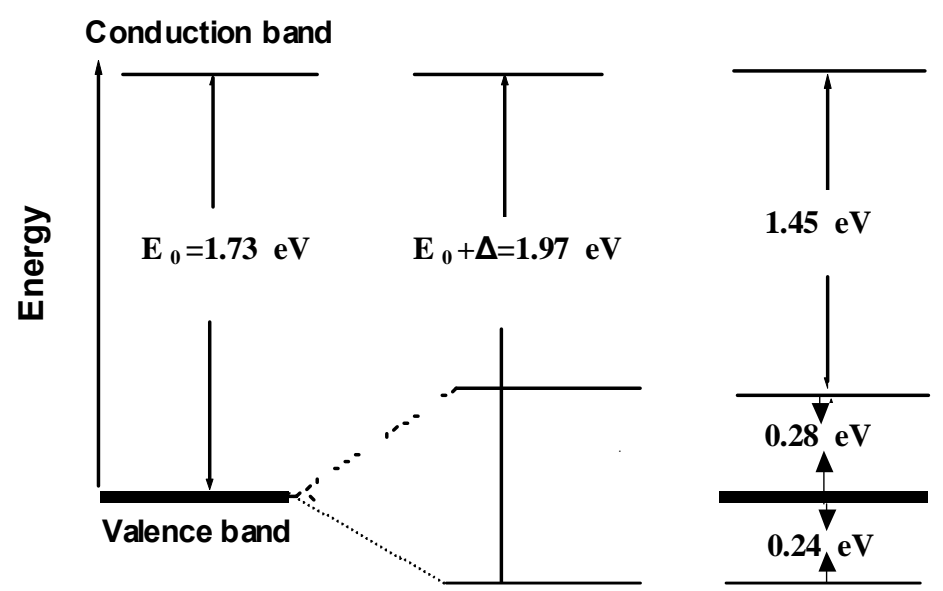

Fig. (2): Shematic representation of valence band spliting showing the possible electronic transitions. $\mathrm{E}_{\mathrm{o}}$ is the non-perturbed energy band gap.

Figure 3 represents a typical variation of the photoresponse with temperature at the fundamental transition $(1.73 \mathrm{eV})$. The curves exhibit three main regimes. Below $100 \mathrm{~K}$, the photocurrent showed a week increase with temperature leading to an activation energy $=0.05 \mathrm{eV}$. In the temperature range $100-200 \mathrm{~K}$, the photoconductivity remains substantitally constant and a quasi plateau region was obtained. A clear decrease of the photocurrent above $200 \mathrm{~K}$ was observed with a characteristic activation energy $=0.12 \mathrm{eV}$. In this latter region the dark conductivity was found to exceed that of photoconductivity. This general behaviour for the temperature dependence of photoconductivity was also obtained at photon energies 1.45 and $1.97 \mathrm{eV}$. Only the larger incident photon energy, the lower the temperature corresponding to maximum photocurrent and the well defined the plateau region. It is likely that in these three regions the recombination mechanisms are the same. The variation of the photocurrent with temperature could be explained in analogues to the two level model containing deep recombination centres and shallow carrier traps [18]. We propose that defects in the present undoped crystal are associated with dislocations or staking falts due to deviation from the ideal CdSe stoichiometry; vacancy; interstitials and antisites (e.g. Cd in Se site). At low temperatures, the increase of photocurrent with temperature is likely to be carried by electrons hopping at deep defect centres with small photoactivation energy (assuming the electrons are the majority carriers). The temperature dependence then comes from the mobility which is lightly activated. At intermediate temperatures, the electron mobility is not expected to vary strongly with temperature but the 
minority carriers (holes) get thermally reactivated from their defect centres into extended valence band, providing additional free carriers. The electron-hole recombination is then enhanced and the lifetime is shortened leading to the observed thermal quenching in the photocurrent. At sufficient high temperatures, the more generated carriers, the more shorting the lifetime which results in a decrease of photocurrent as the temperature is increased. In summary the temperature dependence of photoconductivity indicates that two opposite activation processes are operative in the two temperature regimes. At high temperature, the negative activation energy may indicate capture energy for carriers.

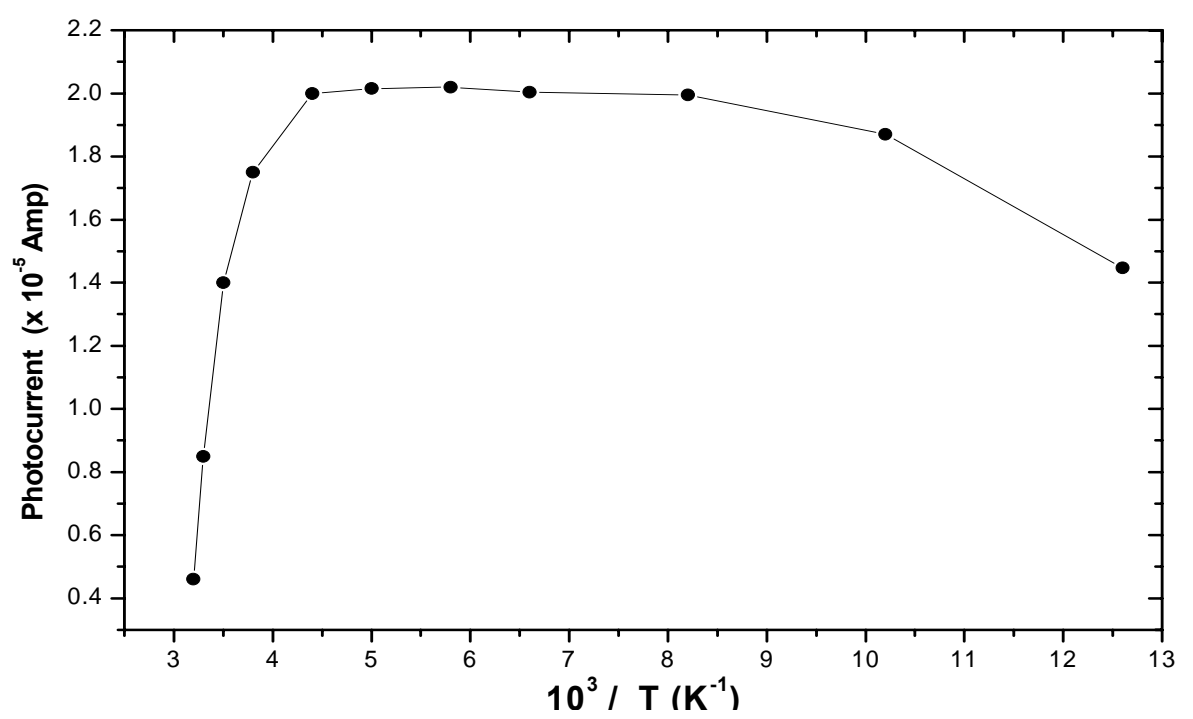

Fig.(3): The photocurrent of the CdSe as a function of temperature, at the fundamental peak $(1.73 \mathrm{eV})$

The dependence of $\Delta \sigma_{\mathrm{ad}} / \Delta \sigma_{\mathrm{st}}$ on the chopping frequency (F), at different temperatures is depicted in Fig.(4). The dependence was found to follow the relation,

$$
\Delta \sigma_{\mathrm{ac}} / \Delta \sigma_{\mathrm{st}}=\operatorname{tansh}(1 / 4 \mathrm{~F} \tau)
$$

where $\Delta \sigma_{\mathrm{ac}} \& \Delta \sigma_{\mathrm{st}}$ stand for the alternating and steady state photoconductivity components. The recombination lifetime $(\tau)$, was obtained directly from the above curves by drawing a parallel line to the frequency axis at a height of 0.76 of the maximum $\Delta \sigma_{\mathrm{ac}} / \Delta \sigma_{\mathrm{st}}$ value (tansh 1=0.76) and dropping a normal from the point of intersection to the frequency axis to cut off a segment at $1 / 4 \tau$ [19]. The measured lifetime has very high values $(1.3-2.5 \mathrm{~ms})$ indicating that the recombination processes are slowed down through defect centres. When plotted 
against temperature, Fig.(5), the lifetime showed three main regimes: almost temperature independent up to $200 \mathrm{~K}$ followed by exponential increase in the range 200-250 $\mathrm{K}$ and rapidly decrease at elevated temperatures. The dependence is explained in accordance to the recombination kinetics as follows: Below $200 \mathrm{~K}$, it is probable that carriers which fall into defect states fairly near the Fermi level do not have ability to escape but recombine from these states. At intermediate temperatures, the dynamics of emission increases with temperature increase and the carriers once getting captured re-excited before recombination and thus the recombination rate decreases. It is likely that the lifetime in this regime depend on the density of defect centres and/or their ability (cross section). Above $250 \mathrm{~K}$, the thermally generated carriers increase (and thereby the recombination rate) leading to a remarkable decrease in the lifetime. In other words the thermal release of carriers into extended states may increase their capturing. The recombination rate in this regime seems to depend on carrier concentration. Such phenomena make the CdSe attractive for the purposes of optoelectronics as a material for the fabrication of photonic devices.

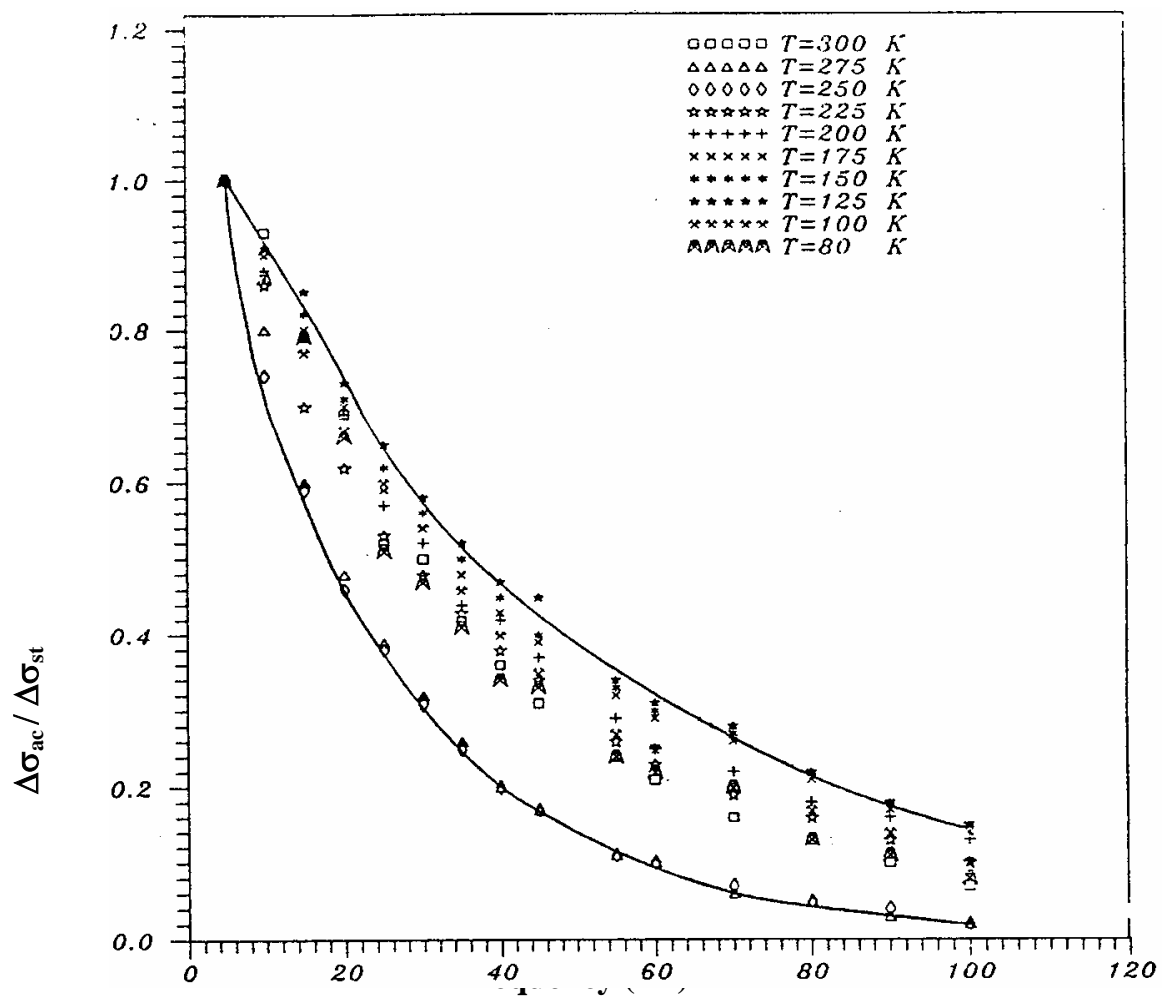

Fig. (4): Kinetics of photoconductivity in CdSe crystal at different temperatures $(80-300 \mathrm{~K})$. 


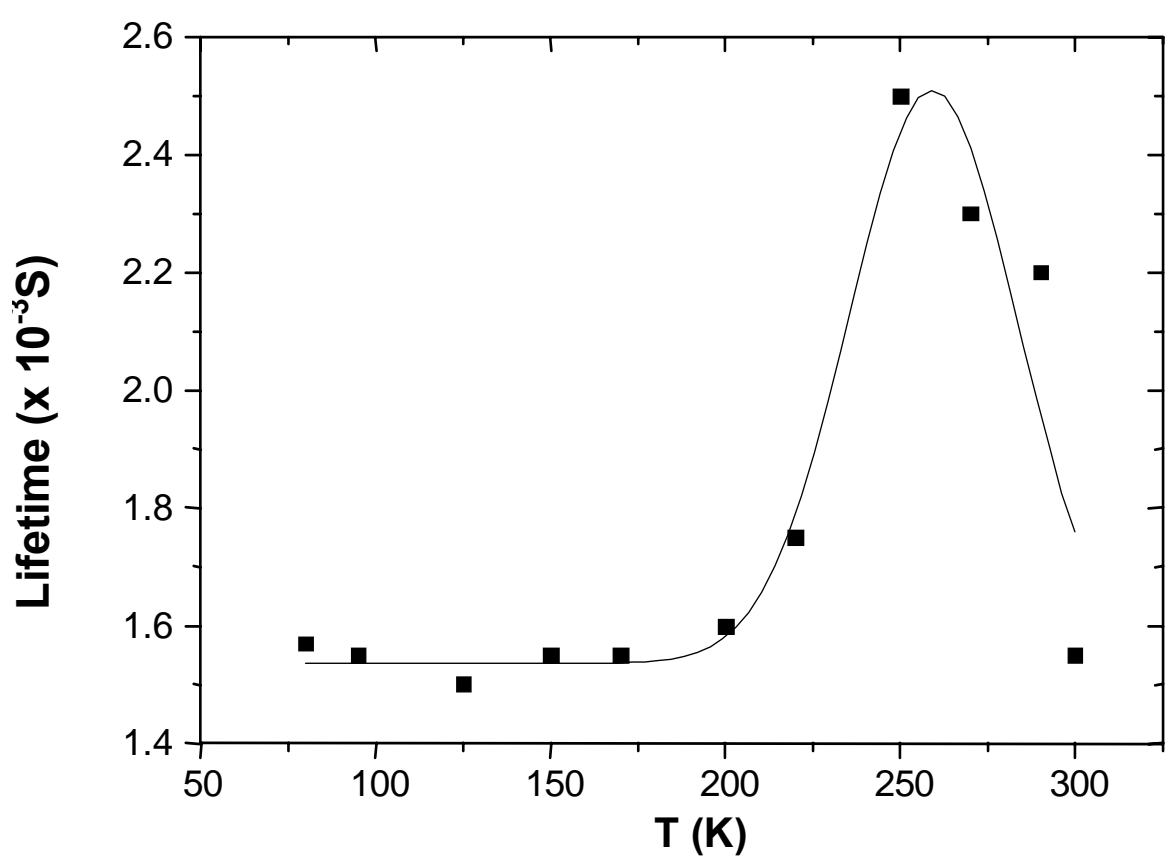

Fig.(5): Temperature dependence of the lifetime in CdSe crystal.

\section{Conclusions:}

Despite the high melting point of CdSe, high quality single crystal is obtained from the classical melt method by adequate temperature gradients and high purity starting material. The peaks in the spectral distribution curves with its temperature dependence bring strong support to the band splitting in the CdSe single crystal. The high lifetime values coupled with its temperature dependence are indicative that native defects are active as capturing centres.

\section{References:}

1. Burger, I. Shilo, M. Schieber, IEEE Trans. Nucl. Sci. 30, 1 (1983) 368.

2. M. Roth and A. Burger, Appl. Phys. Lett. 52, 15 (1988) 1234.

3. R. L. Byer, Photonics Spectra. 25, 1 (1995) 103.

4. J. W. Edmund, E.M. Dale, R. N. Donald, B. E. Arthur, F. G. John, T. F. Kuech, J. Crystal Growth 148 (1995) 63.

5. R. C. Sharma and Y. A. Chang, J. Phase equilibria, 17, 2 (1996) 140.

6. S. Zhu, B. Zhao, Y. Jin. S. Shao, D. Gao, Q. Li, Z. Li, X. Zhu and F.Yu : Cryst. Res. Technol. 35, 11 (2000) 1239.

7. J. S. Yeong and S. J. Tae : New Phys. (Korean Phys. Soci) 31, 6 (1991)784.

8. Siemens, C. Domke and K. Urban : Phys. Rev. B, 59, 4 (1999) 3000. 
9. I. Evtodiev, M. Caraman, M. Petrov : Fizic Starii Condensate p.176 (2000)

10. T. Schmidt, K. Lischkak and W. Zulehner : Phys. Rev. B, 45, 8989 (1992).

11. F. Qusrawi, N. M. Gasanly : Cryst. Res. Technol. 36, 1399 (2000).

12. O. Portillo-Mereno, O. Zelaya-Angel, R. Lozada-Morales, M. RubinFalfan, J. A. Rivera-Marquez : Optical Materials 18, 383 (2002).

13. R. Jenkins, J.L. "DeVries, Practical X-Ray Spectrometry", SpringerVerlag, New York p.22 (1970)

14. A. Ramadan, A. Abd-El Mongy, I. S. Ahmed Farag, A. M. El-Shabiny, F. A. Radwan, H. I. Ismail, H. M. Hashem : Thin Solid films 423, 146 (2003).

15. A Aydinli, N M Gasanly, I Yilmaz and A Serpenguzel : Semicond. Sci. Tecnol. 14, 599 (1999).

16. L. C. Law Yan Voon, M. Willatzen, M. Cardona, N. C. Christensen : Phys. Rev. B 53, 10703 (1996).

17. S. Ninomiya, S. Adachi : J. Appl. Phys. 78, 4681 (1996).

18. A. C. Hovral and W. E. Spear : Phil. Mag. B, 51(1985) 113.

19. Abd El-Mongy, A. Belal, H. El Shaikh and A. El Amin : J. Phys. D, Appl. Phys. 30, 161 (1997). 